TRANSACTIONS OF THE

AMERICAN MATHEMATICAL SOCIETY

Volume 358, Number 5, Pages 1971-1981

S 0002-9947(05)04053-5

Article electronically published on December 20, 2005

\title{
TORUS ACTIONS ON WEAKLY PSEUDOCONVEX SPACES
}

\author{
STEFANO TRAPANI
}

\begin{abstract}
We show that the univalent local actions of the complexification of a compact connected Lie group $K$ on a weakly pseudoconvex space where $K$ is acting holomorphically have a universal orbit convex weakly pseudoconvex complexification. We also show that if $K$ is a torus, then every holomorphic action of $K$ on a weakly pseudoconvex space extends to a univalent local action of $K^{\mathbf{C}}$.
\end{abstract}

\section{INTRODUCTION}

The geometric invariant theory of Mumford and its interpretation in terms of moment maps of hamiltonian actions, is dealing essentially with the holomorphic (algebraic) action of a reductive complex Lie group on a complex space; see [16]. A reductive complex group is the complexification of a compact real Lie group; see 12. Suppose that a complex space $X$ is endowed with an action of a compact Lie group by holomorphic tranformations. In order to study this in the framework of geometric invariant theory, it is convenient to embed $X$ as an open $K$ invariant subset of a good complex space $X^{*}$ with a holomorphic action of the reductive complexified group $K^{\mathbf{C}}$. By the results of Palais [17] and Heinzner-Iannuzzi 11, in a broad variety of situations a canonical space $X^{*}$ as above can be constructed as a non-necessarily Hausdorff complex space. However Heinzner proved in [7] that if $X$ is Stein, then $X^{*}$ exists, is Hausdorff, and in fact it is a Stein space. Later Heinzer and Iannuzzi proved that if $X$ is holomorphically convex, then $X^{*}$ exists, is Hausdorff and is holomorphically convex. Iannuzzi recently dealt in [13] with the case of the action of some non-compact group on taut manifolds. He proved that also in such cases $X^{*}$ exists and it is Hausdorff. In this paper we consider the case of action of compact groups on complex spaces having some weak pseudoconvexity. If the action is good enough we again prove that the space $X^{*}$ exists, it is Hausdorff, and it carries the same weak pseudoconvexity properties of the original space $X$. We also prove that every action of a torus is as we need. In the case $X$ is a Kaeler manifold these weak pseudoconvexity notions are sufficient to prove vanishing theorems for cohomology of holomorphic vector bundles over $X^{*}$; see [4]. Moreover the results of [11] allow, in the hamiltonian situation, to construct suitable quotients. Note that our pseudoconvexity condition seems to be close to optimal, at least in the case of $S^{1}$ actions, to make sure that the Hausdorff property holds for every complexification $X^{*}$. See Remark 3.2. We would like to thank Professor Jean Pierre Demailly for discussions about weak pseudoconvexity.

Received by the editors August 7, 2003 and, in revised form, January 27, 2004.

2000 Mathematics Subject Classification. Primary 32M05, 32U10.

(C)2005 American Mathematical Society Reverts to public domain 28 years from publication 


\section{Preliminaries}

2.1. Local actions. In this subsection we give a quick exposition of the Palais theory in [17] on local actions of Lie groups and on its adaptation to the complex analytic setting given in [11. For more details we refer to the original papers.

Let $X$ be a reduced complex space, and let $G$ be a connected Lie group. A local action of $G$ on $X$ is given by a real analytic map $\Phi: \Omega \rightarrow X$, where $\Omega$ is an open neighborhood of the neutral section $e \times X$ in $G \times X$ with the following properties:

i) For all $x \in X$ the open subset $\Omega_{x}=\{g \in G:(g, x) \in \Omega\}$ is connected.

ii) For fixed $g \in G$ the map $x \rightarrow \Psi(g, x)=g x$ is holomorphic when defined.

iii) For every $g, h \in G$ and $x \in X$ we have $e x=x$, moreover, if $(g h, x) \in$ $\Omega,(h, x) \in \Omega$, and $(g, h x) \in \Omega$, then $(g, h x)=(g h, x)$.

For a complex Lie group the local action is said to be holomorphic if $\Psi$ is holomorphic.

To every local action of $G$ we can associate a linear map $\xi$ from the Lie algebra $\mathrm{g}$ of $G$ to the algebra of holomorphic vector fields on $X$ given by $v \rightarrow \frac{\partial \exp (t v) x)}{\partial t}_{\mid t=0}$. This map is a homomorphism of Lie algebras. Conversely every such homomorphism induces a local action. If $G$ is a complex Lie group, the local holomorphic $G$ action corresponds to $\mathbf{C}$ linear homomorphisms. A product $N \times U$, where $N$ is an open connected neighborhood of $e$ in $G$ and $U$ is an open set of $X$, is called an elementary slice pair if $N=N^{-1}, N^{2} \times U \subseteq \Omega$ and $N^{2} \times N^{2} U \subseteq \Omega$. Given an elementary slice pair $N \times U$ and $h \in G$, we define as a slice chart the map $\Phi: N h \times U \rightarrow G \times X$ defined as $\Phi(g, x)=\left(g, g h^{-1} x\right)$. The map $\Phi$ is then a homeomorphism of $N h \times U$ onto an open set of $G \times X$.

A subset $V$ is a neighborhood of $\left(h_{0}, y_{0}\right)$ for the leaf topology in $G \times X$ if and only if there exists a slice chart $\Phi$ such that $\left(h_{0}, y_{0}\right) \in \Phi\left(N h \times x_{0}\right) \subseteq V$ for some $x_{0} \in U$. A connected component of $G \times X$ in the leaf topology is called a leaf of the local action. If $X$ is smooth, then the leaves are the maximal integral submanifolds of the distribution $\xi(\mathrm{g})$ coming from the diagonal local action of $G$ on $G \times X$ obtained from the left action of $G$ on itself. Let us consider the space

$$
X^{*}=G \times X / \text { modulo leaves }
$$

with the quotient topology. We call $X^{*}$ the Palais globalization of the local action. Note that the leaves are invariant with respect to the diagonal local action. Moreover, the right action of $G$ on the first factor of $G \times X$ induces a global action of $G$ on $X^{*}$. Let $\Pi: G \times X \rightarrow X^{*}$ be the natural projection and let $j: X \rightarrow X^{*}$ be given by $j(x)=\Pi(e, x)$. Then both $\Pi$ and $j$ are continuous open mappings; see 11. Moreover, if $G$ is complex each leaf is a complex locally closed submanifold of $G \times X$, and the restriction to the leaf of the projection on the first factor is a locally biholomorphic map. The local action is said to be univalent if for each leaf the above local biholomorphic map is injective. In other words (since the right action of $G$ on the first factor of $G \times X$ transforms leaves into leaves) the action is univalent if and only if $j$ is injective. For a univalent local action we can use the slice charts to define a holomorphic atlas on $X^{*}$ which makes $X^{*}$ a possibly nonHausdorff complex space. For notational convenience a non-necessarily Hausdorff complex space will be called a quasi-complex space in this paper. The map $j$ then becomes a holomorphic open locally $G$ equivariant embedding. Note that if $Y$ is a quasi-complex space with a global $G$ action the map $(g, y) \rightarrow g^{-1} y$ induces a $G$ equivariant biholomorphism of $Y^{*}$ onto $Y$. We also have the following. 
Lemma 2.1. The space $X^{*}$ has the following universal property. If $Y$ is a quasicomplex space with a holomorphic $G$ action and $f: X \rightarrow Y$ is a locally $G$ equivariant holomorphic map, then there exists a unique holomorphic $G$ equivariant map $f^{*}$ : $X^{*} \rightarrow Y$ such that $f^{*} \circ j=f$. Moreover $G j(X)=X^{*}$.

Proof. See Theorem 2 of section 3 in [11.

Any quasi-complex space with a $G$ action satisfying the condition of the lemma is called a universal globalization of $X$.

A local $G$ action is univalent if and only if it admits some globalization. In such a case it admits a universal globalization, i.e. $X^{*}$, in the category of quasicomplex spaces. There are however examples of spaces with a local $G$ action which admit a universal globalization in the category of Hausdorff complex spaces which is different from $X^{*}$.

We now consider the case where there exists a global $G$ action on the complex space $X$ by holomorphic transformation. Assume moreover that the Lie algebra of $G^{\mathbf{C}}$ is the complexification of the Lie algebra of $G$. This is the case for example if $G$ is compact or simply connected. The global action of $G$ induces a local holomorphic action of the complexified group $G^{\mathbf{C}}$; see 14. In this case we will call $X^{*}$ the complexification of the $G$ action on $X$.

Lemma 2.2. If there exists a global action on $X$ of the group $(\mathbf{R},+)$, then the local $(\mathbf{C},+)$ action is univalent. Similarly if there exists a global action of $S^{1}$, then the local action of $\mathbf{C}^{*}$ is univalent.

Proof. In the case of $(\mathbf{R},+)$ let $F$ be an $(\mathbf{R},+)$ equivariant connected Riemann domain over $\mathbf{C}$; then the action of $(\mathbf{R},+)$ on $F$ is free and proper. The quotient Fmod $\mathbf{R}$ is then a connected non-compact one-dimensional real manifold, and the induced map from Fmod $\mathbf{R}$ into $\mathbf{R}$ is a local diffeomorphism, hence it is injective. Therefore by $(\mathbf{R},+)$ equivariance and freeness the original map is also injective. A similar proof works for the case of $S^{1}$.

Definition 2.3. Let $G$ be a connected Lie group with Lie algebra g. The group $G$ is said to be polar if the map from $G \times \mathbf{g}$ into $G^{\mathbf{C}}$ defined as $(g, v) \rightarrow g \exp (i v)$ is a diffeomorphism.

For example $\left(\mathbf{R}^{n},+\right)$ is polar and every compact connected Lie group is polar.

Definition 2.4. Let $G$ be a real connected polar Lie group with Lie algebra $\mathbf{g}$. Let $G^{\mathbf{C}}$ be the complexification of $G$. Let $Y$ be a quasi-complex space with a $G^{\mathbf{C}}$ action such that $X$ is a $G$ invariant open subset of $Y$ and $G^{\mathbf{C}} X=Y$. We say that $X$ is orbit connected in $Y$ if for every $x \in X$ the set

$$
\Omega_{x}=\left\{g \in G^{\mathbf{C}}: g x \in X\right\}
$$

is connected. We say that $X$ is orbit convex in $Y$ if for every $x \in X$ and $v \in g$ such that $\exp (i v) x \in X$ one has $\exp (i t v) x \in X$ for every $t$ in the interval $[0,1]$.

Lemma 2.5. Let $G$ be a real connected polar Lie group with Lie algebra g. Let $G^{\mathbf{C}}$ be the complexification of $G$. Let $Y$ be a quasi-complex space with a $G^{\mathbf{C}}$ action such that $X$ is a $G$ invariant open subset of $Y$ and $G^{\mathbf{C}} X=Y$. Then $Y$ is $G^{\mathbf{C}}$ equivariantly biholomorphic to the Palais complexification $X^{*}$ of $X$ if and only if $X$ is orbit connected in $Y$.

Proof. See [1]. 
Lemma 2.6. Let $K$ be a compact connected Lie group, and let $F$ be a connected complex manifold with a $K$ action. Let $\pi: F \rightarrow K^{\mathbf{C}}$ be a $K$ equivariant Riemann domain projection (where $K$ acts on $K^{\mathbf{C}}$ by left multiplication). Then $\pi$ is injective if and only if $F$ is holomorphically separated.

Proof. See the proof of Proposition 3 of [11].

Corollary 2.7. Let $X$ be a holomorphically separated complex space, and let $K$ be a compact Lie group acting on $X$ by biholomorphisms. Then the local action of $K^{\mathbf{C}}$ is univalent.

\subsection{P-pseudoconvex spaces.}

Definition 2.8. As in [3] and [15] we say that a complex space $X$ is P-pseudoconvex if it is convex with respect to the family of plurisubharmonic functions, i.e. if for every compact set $H$ the hull $\tilde{H}$ of $H$ with respect to the family of plurisubharmonic functions is relatively compact (note that we consider the family of all plurisubharmonic functions, not only the continuous ones). The complex space $X$ is said to be weakly pseudoconvex if it carries a plurisubharmonic exhaustion function, and for $0 \leq k \leq \infty$ it is $C^{k}$ weakly pseudoconvex if it carries a $C^{k}$ plurisubharmonic exhaustion function.

Note that $X$ is $C^{0}$ weakly pseudoconvex if and only if it is convex with respect to the family of continuous plurisubharmonic functions; see 18 .

Examples 2.9. Every Stein space is $C^{\infty}$ weakly pseudoconvex, more generally by using the Remmert reduction one can see that every holomorphically convex space is $C^{\infty}$ weakly pseudoconvex.

Example 2.10. Let $D$ be a relatively compact domain in a complex manifold $X$ and assume that for a given $k \geq 2$ the domain $D$ has a $C^{k}$ defining function on $X$ which is plurisubharmonic in a neighborhood of $\partial D$; then $D$ is $C^{0}$ weakly pseudoconvex. In 6] Grauert constructs an example of a $D$ as above which is not holomorphically convex.

Example 2.11. Let $X$ be a compact complex manifold and $L$ a holomorphic line bundle on $X$. Let $k \geq 2$ and $h$ be a $C^{k}$ hermitian metric on $L$ with non-negative curvature. Let $D^{*}$ be the set of vectors in the total space of $L^{*}$ having norm smaller than 1 with respect to the dual metric $h^{*}$. Then $D^{*}$ is $C^{0}$ weakly pseudoconvex. Note that, as observed by Grauert, given a holomorphic function $f$ on $D^{*}$, the degree $h$ homogeneous component of the Taylor expansion of $f$ along the fibers gives an holomorphic section of $L^{h}$.

Example 2.12. Let $X$ and $L$ be as above, and assume that $c_{1}(L)$ belongs to the closure of the cone of (Chern classes of ) effective line bundles in $H^{2}(X, \mathbf{R})$. Then $L$ has a semipositive singular metric; see [4]. Then the domain $D^{*}$ defined as above is weakly pseudoconvex. Suppose moreover that the domain $D^{*}$ admits some non-constant holomorphic function, hence for some $k$ the bundle $L^{k}$ admits some non-trivial holomorphic section $s$. We can consider on $L$ the singular metric $h=\left(\frac{1}{\sum_{j}\left|\theta\left(s_{j}\right)\right|^{2}}\right)^{1 / k}$, where $\theta$ is a local trivialization, and $s_{1}, \ldots, s_{N}$ is a base of $H^{0}\left(X, L^{k}\right)$. So $D^{*}$ is $C^{0}$ weakly pseudoconvex. 
Lemma 2.13. Every weakly pseudoconvex Riemann domain $X$ over a Stein space $Y$ is Stein. Moreover, if $Y$ is smooth and $X$ is only P-pseudoconvex, then it is Stein.

Proof. Let $Y$ be a Stein space with a Riemann domain projection $\pi: X \rightarrow Y$. Let $\phi$ a plurisubharmonic exhaustion of $X$, and let $\alpha$ be a smooth strictly plurisubharmonic function on $Y$. The function $\phi+\exp (\alpha \circ \pi)$ is a strictly plurisubharmonic exhaustion of $X$. By 2 the space $X$ is holomorphically convex. Since however $\alpha \circ \pi$ is a smooth strictly plurisubharmonic function of $X$, the space $X$ cannot contain positive-dimensional compact analytic subsets. Hence $X$ is Stein. Assume now that $Y$ is smooth and $X$ is $\mathrm{P}$-pseudoconvex. If we consider $Y$ as a closed analytic subvariety of some $\mathbf{C}^{N}$ we can find a domain of holomorphy $D$ in $\mathbf{C}^{N}$ containing $Y$ and a holomorphic retraction $\rho: D \rightarrow Y$. That is, $\rho$ is holomorphic and $\rho_{\mid Y}=I d_{\mid Y}$. Let $Z$ be the fiber product of $(X, \pi)$ and $(D, \rho)$. Then $Z$ is a closed submanifold of $X \times D$, hence it is a P-pseudoconvex, and the projection on the second factor makes it a Riemann domain over $D$. Moreover, $X$ is biholomorphic to the closed analytic subvariety $Z \cap(X \times Y)$ of $Z$. It follows easily from the maximum principle for plurisubharmonic functions that the manifold $Z$ satisfies the Oka "Kontinuitatssatz" condition, hence $Z$ is Stein and so is $X$. See [19].

Lemma 2.14. If $X$ is a complex space with a holomorphic action of a compact Lie group $K$ and $H$ is a compact $K$ invariant subset of $X$, then the hull of $H$ with respect to the set of plurisubharmonic functions on $X$ coincides with the hull with respect to the set of $K$ invariant ones.

Proof. The hull with respect to the plurisubharmonic functions is clearly contained in the hull with respect to the $K$ invariant once. Now to every plurisubharmonic function $\phi$ on $X$ we associate the plurisubharmonic $K$ invariant function $\psi(x)=$ $\max _{K} \phi(k x)$. Then $\phi \leq \psi$, and if $H$ is a $K$ invariant compact subset of $X$, then $\max _{H} \phi=\max _{H} \psi$. Therefore if $x$ belongs to the hull of $H$ with respect to the $K$ invariant plurisubharmonic function, then $\phi(x) \leq \psi(x) \leq \max _{H} \psi=\max _{H} \phi$.

Lemma 2.15. Let $Y$ be a Stein space with a holomorphic $K$ action, and let $\phi$ be a plurisubharmonic $K$ invariant function of $X$. Then for every $x \in X$ and every vector $\xi$ in the Lie algebra of $K$, the function $\Phi(t)=\phi(\exp (i t \xi x))$ is convex in $t$.

Proof. The result is known in the case where $\phi$ is smooth; see [7. In the general case, since $Y$ is Stein we know from [5] that there is a sequence $\phi_{n}$ of smooth strictly plurisubharmonic functions on $Y$ pointwise decreasing to $\phi$. By integration on the $K$ orbits and using Dini theorem, we find a sequence of smooth strictly plurisubharmonic $K$ invariant functions pointwise decreasing to $\phi$. The result follows.

\section{MAIN THEOREM}

Theorem 3.1. Let $X$ be a P-pseudoconvex space, and let $K$ be a compact connected Lie group acting on $X$ by biholomorphisms. Assume that the local action of $K^{\mathbf{C}}$ is univalent. Then the Palais globalization $X^{*}$ of $X$ is a Hausdorff complex space. Moreover, $X$ is orbit convex in $X^{*}$, and the holomorphic functions on $X$ can be approximated by holomorphic functions on $X^{*}$ in the compact-open topology. If $X$ is weakly pseudoconvex or $C^{0}$ weakly pseudoconvex, then so is $X^{*}$. Moreover, if $X$ is smooth and $C^{k}$ weakly pseudoconvex with $k \geq 1$, then so is $X^{*}$. 
Proof. Since the local action of $K^{\mathrm{C}}$ is assumed to be univalent, we know that the space $X^{*}$ exists as a quasi-complex space. Moreover, the leaves of the Palais foliation in $K^{\mathbf{C}} \times X$ are closed subspaces. On the other hand $K^{\mathbf{C}}$ is a Stein manifold (see [8]), therefore $K^{\mathrm{C}} \times X$ is P-pseudoconvex and so is every leaf of the foliation. By Lemma 2.13 every leaf is therefore Stein. By univalence the image of the leaf by the projection on the first factor is also Stein. Hence by 7 for every $x \in X$ the set $\Omega_{x}=\left\{g \in K^{\mathbf{C}}: g x \in X\right\}$ is orbit convex in $K^{\mathbf{C}}$, that is to say that $X$ is orbit convex in $X^{*}$. Let $\eta$ be a vector in the Lie algebra of $K$. Consider the action of $(\mathbf{R},+)$ on $X$ given by $t * x=\exp (t \eta) x$. By Lemma 2.2 the local action of $\mathbf{C}$ is univalent. Let $X_{\eta}$ be the Palais globalization of $X$ with respect to this action. Since $X$ is orbit convex in $X^{*}$ it follows from Lemma 2.5 that each $X_{\eta}$ can be identified with an open set of $X^{*}$. Moreover, since $K$ is polar, given two points $p, q$ in $X^{*}$, there exists an element $g \in K^{\mathbf{C}}$ such that $g p$ belongs to $X$ and $g q$ belongs to some $X_{\eta}$. Therefore to prove that $X^{*}$ is Hausdorff is equivalent to proving that each $X_{\eta}$ is Hausdorff. Fix $\eta$ in the Lie algebra of $K$ and consider the associated action of $(\mathbf{R},+)$ as above. Assume by contradiction that $X_{\eta}$ is not Hausdorff. Let $x_{1}$ and $x_{2}$ be two distinct points in $X_{\eta}$ which have no disjoint neighborhoods. Assume $x_{1} \in X$. Let $\Delta_{x_{k}}=\left\{t \in \mathbf{R}: i t * x_{k} \in X\right\}$, with $k=1,2$. If there existed $t_{0} \in \Delta_{x_{1}} \cap \Delta_{x_{2}}$, then we would have $i t_{0} * x_{1} \in X$ and $i t_{0} * x_{2} \in X$. Since $X$ is Hausodorff and open in $X_{\eta}$ this is not possible. So $\Delta_{x_{1}} \cap \Delta_{x_{2}}=\emptyset$. Since the Palais complexification is always orbit connected, we have $\left.\Delta_{x_{1}}=\right] \alpha, \beta\left[\right.$ and $\left.\Delta_{x_{2}}=\right] \gamma, \delta[$. We can assume $\alpha<0<\beta \leq \gamma<\delta$. Let $\left.t_{1} \in\right] \gamma, \delta\left[\right.$. Since $x_{1}$ and $x_{2}$ have no disjoint neighborhood, there exists a sequence $p_{n}$ in $X_{\eta}$ such that $p_{n}$ converges to $x_{1}$ and $p_{n}$ converges to $x_{2}$ so that for large enough $n_{0}$ we have that $p_{n_{0}+n}$ converges to $x_{1}$ and $i t_{1} * p_{n_{0}+n}$ converges to $i t_{1} * x_{2}$ in $X$. Since $X$ is orbit connected in $X_{\eta}$ we know that $i \beta * p_{n_{0}+n}$ is in $X$. Now let $H \subseteq X$ be defined by

$$
H=K\left(\bigcup_{n}\left(p_{n_{0}+n}\right) \cup\left(x_{1}\right) \bigcup_{n}\left(i t_{1} * p_{n_{0}+n}\right) \cup\left(i t_{1} * x_{2}\right)\right) .
$$

Then $H$ is a compact $K$ invariant subset of $X$. Let $\phi$ be a plurisubharmonic $K$ invariant function on $X$, and let $M$ be the maximum of $\phi$ in $H$. Let us fix $n \geq 0$ and restrict $\phi$ to the Stein local $\mathbf{C}$ orbit of $p_{n_{0}+n}$. It follows that the function $\Psi(t)=\phi\left(\exp (i t \eta) p_{n_{0}+n}\right)=\phi\left(i t * p_{n_{0}+n}\right)$ is convex. Since $\Psi(0) \leq M$ and $\Psi\left(t_{1}\right) \leq M$ we have $\Psi(\beta)=\phi\left(i \beta * p_{n_{0}+n}\right) \leq M$. In other words, by Lemma 2.2 the point $i \beta * p_{n_{0}+n}$ lies in $\tilde{H}$. Since $\tilde{H}$ is relatively compact, the sequence $i \beta p_{n_{0}+n}$ must have a subsequence which converges in $X$. So we can assume, by renaming the sequence, that $i \beta * p_{n}$ converges to $\tilde{x}$ in $X$. For small positive $\varepsilon$ we then have that $i(\beta-\varepsilon) * p_{n}$ converges to $-i \varepsilon * \tilde{x}$ in $X$. However $i(\beta-\varepsilon) * p_{n}$ converges to $i(\beta-\varepsilon) * x_{1}$ in $X$. Therefore $i \beta * x_{1}=\tilde{x} \in X$, and since $X$ is open in $X^{*}$ for small positive $\varepsilon$, we also have that $i(\beta+\varepsilon) * x_{1} \in X$. This is in contrast with the definition of $\Delta_{x_{1}}$. So we have found a contradiction. Therefore $X^{*}$ is an actual Hausdorff complex space.

The Fourier series expansion, together with the universal property of $X^{*}$, proves that the holomorphic functions on $X^{*}$ are dense in the holomorphic functions on $X$ in the compact open topology; see [7]. We are left to show that if $X$ is weakly pseudoconvex, then so is $X^{*}$. Let $\phi$ be a positive plurisubharmonic exhaustion of $X$. By averaging we can assume that $\phi$ is $K$ invariant. Since $K$ is compact if the original $\phi$ is an exhaustion, the averaged one is also an exhaustion. The group $K^{\mathrm{C}}$ 
is a Stein manifold; let $\rho$ be a positive smooth $K$ invariant strictly plurisubharmonic exhaustion in $K^{\mathbf{C}}$. The function $\tau(g, x)=\rho(g)+\phi(x)$ is a plurisubharmonic exhaustion function on $K^{\mathbf{C}} \times X$. Let $\tau^{*}: X^{*} \rightarrow \mathbf{R} \cup\{-\infty\}$ be defined by

$$
\tau^{*}(p)=i n f_{(g, x) \in \Pi^{-1}(p)} \tau(g, x) .
$$

Here $\Pi: K^{\mathbf{C}} \times X \rightarrow X^{*}$ is the natural projection. Since $\Pi$ is an open mapping, $\tau^{*}$ is easily seen to be an upper semicontinuous exhaustion function. We need to show that it is plurisubharmonic. Consider the map $F: K^{\mathbf{C}} \times X \rightarrow K^{\mathbf{C}} \times X^{*}$ defined by $F(g, x)=(g, \Pi(g, x))$; then $F$ is holomorphic and injective. Moreover, the set $\Omega=F\left(K^{\mathrm{C}} \times X\right)$ is given by $\Omega=\{(g, p): g p \in X\}$. So $\Omega$ is open and $F^{-1}: \Omega \rightarrow K^{\mathbf{C}} \times X$ is the map $F^{-1}(g, p)=(g, g p)$. Therefore $F$ is a holomorphic embedding and the diagonal action of $K$ on $K^{\mathbf{C}} \times X$ transforms via $F$ in the left multiplication on the first factor in $K^{\mathbf{C}} \times X^{*}$. Moreover, the leaves of the Palais foliation transform via $F$ in the fibers of the restriction to $\Omega$ of the projection $P_{2}$ on the second factor. From now on we identify $K^{\mathbf{C}} \times X$ with $\Omega$.

Let $U$ be a Stein neighborhood of $p$ in $X^{*}$. Then $\Omega \cap\left(K^{\mathbf{C}} \times U\right)$ is a weakly pseudoconvex open subset of a Stein space, hence it is Stein. By [5], there exists a decreasing sequence $\tau_{n}$ of smooth strictly plurisubharmonic functions which pointwise converge to $\tau$ on $\Omega \cap\left(K^{\mathbf{C}} \times U\right)$. By averaging we may assume that each $\tau_{n}$ is $K$ invariant. (Since $\tau$ is constant on the $K$ orbits, by Dini theorem this averaged $\tau_{n}$ is still pointwise converging to $\tau$.) Note that for each fixed $n$ the map $\tau_{n}$ is an exhaustion on $\Omega$. Therefore the map $\tau_{n} \times P_{2}: \Omega \cap\left(K^{\mathbf{C}} \times U\right) \rightarrow \mathbf{R} \times U$ is proper. From [10] we know that the functions

$$
\tau^{*}{ }_{n}(p)=i n f_{(g, p) \in \Omega} \tau_{n}(g, p)
$$

are strictly plurisubharmonic in $U$. On the other hand $\tau^{*}{ }_{n}$ is pointwise decreasing to $\tau_{*}$, hence $\tau_{*}$ is plurisubharmonic.

Assume now that $\phi$ is continuous, hence $\tau$ is also continuous. Since $\tau$ is an exhaustion, the infimum over the leaves is a minimum, so that $\tau^{*}$ will never take the value $-\infty$. Moreover, $\tau^{*}$ is upper semicontinuous, i.e. for every $p \in X^{*}$, we have $\tau^{*}(p) \geq \lim \sup _{x \rightarrow p} \tau^{*}(x)$. Now let $p_{n} \in X^{*}$ converge to $p$ with $\tau^{*}\left(p_{n}\right)$ converging to $l=\liminf _{x \rightarrow p} \tau^{*}(x)$. Since $\tau$ is a continuous exhaustion, there exists $q_{n} \in \Omega$ with $P_{2}\left(q_{n}\right)=p_{n}$ and $\tau\left(q_{n}\right)=\tau^{*}\left(p_{n}\right)$. Since for large $n$ we have $\tau\left(q_{n}\right)<l+1$, up to subsequence (which we can rename as above) we may assume that $q_{n}$ converges to $q_{\infty} \in \Omega$ with $P_{2}\left(q_{\infty}\right)=p$. By continuity of $\tau$ we have $l=\tau\left(q_{\infty}\right) \geq \tau^{*}(p)$. Hence $\tau^{*}$ is continuous.

If $X$ is smooth, then so is $X^{*}$. If $\phi$ is $C^{k}$ with $k \geq 2$, then so is $\tau$. To show that $\tau^{*}$ is $C^{k}$ we proceed as in 9]. Note that $\tau$ is strictly plurisubharmonic along the fibers of $P_{2}$. In particular, in every fiber of $P_{2}$ over $U$ the function $\tau$ has a unique $K$ orbit of critical points, and they are absolute minima. On the other hand, given a smooth strictly plurisubharmonic function $\Psi$ on $U$, we find that the function $\Theta=\tau+\Psi \circ P_{2}$ is $C^{k}$ and strictly plurisubharmonic on $Y=\left(K^{\mathbf{C}} \times U\right) \cap \Omega$. To the two forms $\omega_{\tau}=2 i \partial \bar{\partial} \tau$ and $\omega_{\Theta}=2 i \partial \bar{\partial} \Theta$ we associate the corresponding moment maps $\mu_{\tau}(x, \xi)=d \tau_{x}\left(J \xi_{Y}\right)$ and $\mu_{\Theta}(x, \xi)=d \Theta_{x}\left(J \xi_{X}\right)$. Here $J$ is the complex structure, $\xi$ is a vector in the Lie algebra of $K$, and $\xi_{Y}$ is the vector field associated to $\xi$ via the action of $K$ on $K^{\mathbf{C}} \times U$. See for example 9]. However $\mu_{\tau}=\mu_{\Theta}$ and $\omega_{\Theta}$ is a Kaeler form. Since $X$ is smooth and the $K$ action is free, it follows that the set $R=\mu_{\tau}^{-1}(0)=\mu_{\Theta}{ }^{-1}(0)$ is a $C^{k-1}$ submanifold and coincides with the set of minima of $\tau$ and $\Theta$ along the fibers of $P_{2}$. We can choose local coordinates 
$(z, w)$ around a point $(g, p)$ in $K^{\mathbf{C}} \times U$ so that $z=x+i y$ and the vectors $\frac{\partial}{\partial x_{\alpha}}$ are tangent to the $K$ orbits. We think of $\tau$ and $\Theta$ as functions of $y$ and $w$ only. There is a $C^{k-1}$ function $y(w)$ defined in a neighborhood of $p$ such that, for $w$ in such a neighborhood, $(i y(w), w) \in R / K$. This means that $\tau^{*}(w)=\tau(i y(w), w)$ is $C^{k-1}$. To show that $\tau^{*}$ is in fact $C^{k}$ we note that $R / K$ is the zero set of $\frac{\partial \tau}{\partial y}$. It follows that $\frac{\partial \tau^{*}}{\partial w}=\frac{\partial \tau}{\partial w}(i y(w), w)$ is $C^{k-1}$, hence $\tau^{*}$ is $C^{k}$.

Suppose now that $k=1$. As in the proof of Lemma 2.13 we choose a holomorphic retraction $r: D \rightarrow Y=\left(K^{C} \times U\right) \cap \Omega$, where $D$ is a domain of holomorphy in $\mathbf{C}^{N}$ and $Y$ is considered as a closed submanifold of $\mathbf{C}^{N}$. If $\phi$ is $C^{1}$, then so is $\tilde{\phi}=\phi \circ r$. Let us consider the usual smoothing $\tilde{\phi}_{\varepsilon}$ of $\tilde{\phi}$ by convolution. Then, when $\varepsilon$ goes to zero, $\tilde{\phi}_{\varepsilon}$ converges to $\tilde{\phi}$ in $C^{1}$ norm on compact sets. Set $\tilde{\tau}=\tau \circ r$ and $\tilde{\Theta}=\Theta \circ r$. Note that, since $\tilde{\phi}$ is plurisubharmonic and $\rho$ is smooth and strictly plurisubharmonic in $K^{\mathbf{C}}$, then if $\omega$ is a positive smooth $(1,1)$ form on $Y$, then

$$
\frac{\partial \tilde{\Theta}_{\varepsilon}}{\partial z \partial \bar{z}}>\lambda \omega\left(\frac{\partial}{\partial z}, \frac{\partial}{\partial \bar{z}}\right)
$$

on every compact subset. Here $\lambda$ is a positive real number depending only on the compact subset chosen. It follows that the solution $y_{\varepsilon}(w)$ of the equation

$$
\frac{\partial \tilde{\Theta}_{\varepsilon}}{\partial y}\left(i y_{\varepsilon}(w), w\right)=0
$$

has first derivatives uniformly bounded on compact sets with respect to $\varepsilon \rightarrow 0$. On the other hand

$$
\Theta\left(i y_{\varepsilon}(w), w\right) \leq \tilde{\Theta}_{\varepsilon}\left(i y_{\varepsilon}(w), w\right) \leq \tilde{\Theta}_{\varepsilon}(0, w) \leq \Theta(0, w)+1<C,
$$

for small $\varepsilon$, large $C$ and $w$ in a relatively compact subset of $U$. Since $\Theta$ is an exhaustion, the functions $y_{\varepsilon}(w)$ are also uniformly bounded on compact sets of $U$ as $\varepsilon \rightarrow 0$. Therefore there exists a sequence $\varepsilon_{k} \rightarrow 0$ such that $y_{\varepsilon_{k}}$ converges uniformly on compact subsets of $U$ to a continuous function $y(w)$. It follows from uniform convergence of $\tilde{\tau}_{\varepsilon}$ that $\tau(i y(w), w)=\tau^{*}(w)$. On the other hand, because of uniform $C^{1}$ convergence of $\tilde{\tau}_{\varepsilon}$, each derivative

$$
\frac{\partial \tilde{\tau}_{\varepsilon_{k}}^{*}}{\partial w_{s}}(w)=\frac{\partial \tilde{\tau}_{\varepsilon_{k}}}{\partial w_{s}}\left(i y_{\varepsilon_{k}}(w), w\right)
$$

also converges uniformly on a compact set. Therefore $\tau^{*}$ is $C^{1}$ and

$$
\frac{\partial \tau^{*}}{\partial w_{s}}(w)=\frac{\partial \tau}{\partial w_{s}}(i y(w), w) .
$$

Remark 3.2. With a proof as above one can show the following: Let $K$ be a compact connected Lie group so that $K^{\mathrm{C}}$ is acting locally and in a univalent way on the complex space $X$. Assume that there exists on $K^{\mathbf{C}} \times X$ an exhaustion function which is plurisubharmonic when restricted to each leaf of the Palais foliation; then the Palais complexification is Hausdorff. Moreover, in the case of the group $S^{1}$ acting on a smooth manifold, we can reverse this statement. More precisely, if $X$ is smooth, $K=S^{1}$ and $X^{*}$ is Hausdorff, then there exists a continuous exhaustion on $\mathbf{C}^{*} \times X$ which is subharmonic when restricted to each leaf of the Palais foliation. 
Theorem 3.3. Let $X$ be a $C^{\infty}$ weakly pseudoconvex complex Kaeler manifold, and let $K$ be a compact connected Lie group acting on $X$ by biholomorphisms. Assume that the local action of $K^{\mathbf{C}}$ is univalent. Then $X^{*}$ is also a Kaeler manifold.

Proof. As in the proof of the main theorem we can identify $K^{C} \times X$ with the subset $\Omega$ of $K^{C} \times X^{*}$ given by $\Omega=\left\{(g, p) \in K^{C} \times X^{*}\right.$ such that $\left.g p \in X\right\}$. Here the identification is $(g, x) \rightarrow\left(g, g^{-1} x\right)$. Now let $\phi$ be a smooth plurisubharmonic $K$ invariant exhaustion of $X$. Let $\Psi$ be a smooth strictly plurisubharmonic $K$ invariant exhaustion of $K^{C}$. Then $\phi+\Psi$ is a smooth plurisubharmonic exhaustion of $K^{C} \times X$, which induces a smooth plurisubharmonic exhaustion $\phi^{\prime}$ of $\Omega$. Finally let $\hat{\phi}=\phi^{\prime}+\Psi$. Then $\hat{\phi}$ is a smooth plurisubharmonic $K$ invariant exhaustion on $\Omega$ which is strictly plurisubharmonic in the $K^{\mathbf{C}}$ directions. Let $\Sigma$ be the union over $p \in X^{*}$ of the critical sets of $\hat{\phi}$ restricted to $K^{\mathbf{C}} \times\{p\}$. Then we see as in the proof of the main theorem that $\Sigma$ is a closed real submanifold of $\Omega$ and that the restriction to $\Sigma$ of the projection $K^{\mathbf{C}} \times X^{*} \rightarrow X^{*}$ is of maximal rank surjective and with $K$ orbits as fibers. Now let $\omega$ be a Kaeler form on $X$. Then $i \partial \bar{\partial} \Psi+\omega$ induces a $K$ invariant Kaeler form $\tilde{\omega}$ on $\Omega$. Let $h$ be the corresponding Riemannian metric. Then for $(g, p) \in \Sigma$ the intersection $T_{(g, p)} \Sigma \cap(K(g, p))^{\perp}{ }_{h}$ coincides with the complex tangent space $T^{\mathbf{C}_{\Sigma_{(g, p)}}}=T_{(g, p)} \Sigma \cap J T_{(g, p)} \Sigma$ of $\Sigma$ at $(g, p)$ (here $J$ is the complex structure of $\Omega$ ). This space is identified with the tangent space of $X^{*}$ at $p$ via the differential of the projection on the second factor. This induces a Kaeler metric on $X^{*}$.

Definition 3.4. Let $E$ be an holomorphic vector bundle over a complex $n$ dimensional manifold $Y$, and let $s$ be an integer with $1 \leq s \leq n$. The vector bundle $E$ is said to be $s$ positive if it has a smooth hermitian metric whose curvature is positive when evaluated on non zero tensors of rank not greater then $s$. (The 1 positivity is the Griffith positivity, whereas the $n$ positivity is the Nakano positivity.)

Corollary 3.5. Let $K$ be a compact connected Lie group. Let $K^{\mathbf{C}}$ be the complexification of $K$. Let $X$ be a $C^{\infty}$ weakly pseudoconvex Kaeler manifold with a $K$ action such that the local $K^{\mathbf{C}}$ action is univalent. Then both $X$ and $X^{*}$ admit complete Kaeler metrics. Let $E$ be an s positive vector bundle over $X^{*}$; then $H^{n, q}\left(X^{*}, E\right)=0$ for $q \geq n-s+1$.

Proof. The existence of complete Kaeler metrics on $X$ and $X^{*}$, as well as the vanishing theorem, follow from the results in [4] and Theorem 3.3

\section{UNIVALENCE CRITERIA}

Besides the general criterion for univalence in Corollary 2.7, we have in the case of P-pseudoconvex spaces the following criteria:

Proposition 4.1. Let $K$ be a compact connected Lie group. Let $K^{\mathrm{C}}$ be the complexification of $K$. Let $Y$ be a quasi-complex space with a $K^{\mathrm{C}}$ action. Let $X$ be a $P$-pseudoconvex space with an action of $K$, and assume that there exists a locally injective holomorphic $K$ equivariant map $f: X \rightarrow Y$. Then the local $K^{\mathbf{C}}$ action on $X$ is univalent.

Proof. Let $f^{*}: X^{*} \rightarrow Y$ be the map induced by $f$ as in Lemma2.1. Since $f=f^{*} \circ j$ is locally injective, so is $j$. Since $j$ is an open mapping and $X^{*}=K^{\mathbf{C}} X$, then the space $X^{*}$ becomes locally euclidean. In particular $X^{*}$ is locally Hausdorff, hence 
each point is closed, i.e. each leaf in $K^{\mathrm{C}} \times X$ is closed. Therefore each leaf is P-pseudoconvex. By Lemma 2.13 each leaf is Stein, and by Lemma 2.6 the local action of $K^{\mathrm{C}}$ is univalent.

Proposition 4.2. Let $Y$ be a P-pseudoconvex space, and let $K$ be a compact connected Lie group so that $K$ is acting holomorphically and $K^{\mathrm{C}}$ is acting locally in a univalent way on $Y$. Let $X$ be a complex space with an action of $K$. Let $\phi: X \rightarrow Y$ be a proper holomorphic $K$ equivariant map. Then $X$ is P-pseudoconvex, and the local action of $K^{\mathrm{C}}$ on $X$ is univalent.

Proof. Clearly $X$ is P-pseudoconvex. Moreover, because of the above theorem the Palais globalization $Y^{*}$ of $Y$ with respect to the local action of $K^{\mathrm{C}}$ exists and $Y$ is orbit convex in $Y^{*}$. The result then follows from the theorem in section 6 of [11.

Proposition 4.3. Let $X$ be a P-pseudoconvex space, and let $T$ be a torus acting on $X$ by biholomorphisms. Then the local action of $T^{\mathbf{C}}$ is univalent.

Proof. We prove the claim by induction on the dimension $n$ of the torus. If $n=1$ the result follows from Lemma 2.2. If $n>1$ we write $T=T^{\prime} \times S^{1}$, where $T^{\prime}$ is an $(n-1)$-dimensional torus. By inductive hypothesis we can construct the Palais complexification $X^{\prime}$ of $X$ with respect to the action of $T^{\prime}$. Since $T$ is commutative, the action of $S^{1}$ on $X$ is $T^{\prime}$ equivariant. By universality this action extends to an action of $S^{1}$ on $X^{\prime}$. Since $X$ is P-pseudoconvex then $X^{\prime}$ is an actual complex space. Then by Lemma 2.2 the Palais globalization $X^{\prime \prime}$ of $X^{\prime}$ with respect to the $S^{1}$ action exists. It is easy to see that $X^{\prime \prime}$ is a complexification of $X$ with respect to the action of $T$. Therefore by Proposition 4.1 the local action of $T^{\mathbf{C}}$ is univalent.

Because of the results of 11 we have existence of Hausdorff complexification of principal bundles over a P-pseudoconvex space $X$ with an equivariant $K$ action. Moreover, again because of 11] we have existence of categorical quotients of hamiltonian $K$ actions on a P-pseudoconvex space $X$.

\section{REFERENCES}

[1] E. Casadio Tarabusi, A. Iannuzzi, S. Trapani, Globalizations, fiber bundles and envelopes of holomorphy Math Z. 233 (2000) pag. 535-551. MR.1750936 (2001f:32035)

[2] M. Coltoiu, N. Mihalache, Strongly plurisubharmonic exhaustion functions on 1-convex spaces Math. Ann. 270 (1985), pag. 63-68. MR0769607 (86a:32037)

[3] J. P. Demailly, Estimations $L^{2}$ pour l'operate'ur $\bar{\partial}$ d'un fibre' vectoriel holomorphe semipositif au dessus d'une variete' Kaelerienne complete Ann. Sci. Ecole Norm. Sup. 15 (1982) pag. 457-511. MR0690650 (85d:32057)

[4] J. P. Demailly, Singular Hermitian metrics on positive line bundles in "Complex algebraic varieties" Bayreuth 1990 (K. Hulek et al., eds.), Lecture Notes in Math. vol. 1507 Springer Berlin (1992) 361-409. MR1178721 (93g:32044)

[5] J. E. Fornaess, R. Narashimhan, The Levi problem on complex spaces with singularities Math. Ann. 248 (1980) pag. 47-72. MR0569410 (81f:32020)

[6] H. Grauert, Bemerkenswerte pseudokonvexe Mannigfaltigkeiten Math Z. 81 (1963) pgs. 377391. MR0168798 (29:6054)

[7] P. Heinzner, Geometric invariant theory of Stein Spaces Math. Ann. 289 (1991) pag. 631-662. MR.1103041 (92j:32116)

[8] P. Heinzner, Equivariant holomorphic extension of real analytic manifolds Bull. Soc. Math. France 121 (1993) pag. 445-463. MR1242639(94i:32050)

[9] P. Heinzner, The minimum principle from an Hamiltonian point of view Doc. Math. 3 (1998) pag. 1-14 (electronic) MR.1620612 (99c:32044) 
[10] P. Heinzner, A. Huckleberry, F. Loose, Kahlerian extension of the symplectic reductions J. Reine Angew. Math. 455 (1994) pag. 123-140. MR.1293876 (95k:58061)

[11] P. Heinzner, A. Iannuzzi, Integration of local actions on holomorphic fiber spaces Nagoja Math. J. 146 (1997) pag. 31-53. MR1460953 (98k:32047)

[12] G. Hochschild, The structure of Lie groups Holden-Day, San Francisco, London, Amsterdam (1965) MR.0207883(34:7696)

[13] A. Iannuzzi, Induced local actions on taut and Stein manifolds preprint.

[14] W. Kaup, Infinitesimale Tranformationengruppen komplexes Math. Ann. 160 (1965) pag. 72-93. MR0181761 (31:5988)

[15] S. Kobayashi, Hyperbolic complex spaces Springer-Verlag (1998). MR1635983 (99m:32026)

[16] D. Mumford, J. Fogarty, F. Kirwan, Geometric Invariant theory Springer-Verlag (Third enlarged edition 1994). MR.1304906 (95m:14012)

[17] R. S. Palais, A global formulation of the Lie theory of transformation groups Mem. of the Amer. Math. Soc. (1955). MR0121424 (22:12162)

[18] Y. T. Siu Holomorphic fiber bundles whose fiber are bounded Stein domains with zero first Betti number Math. Ann. 219 (1976) pgs. 171-192. MR0390303(52:11129)

[19] Y. T. Siu Pseudoconvexity and the problem of Levi Bull. Am. Math. Soc. 84 (1978) pgs. 481-512. MR0477104 (57:16648)

Dipartimento di Matematica, Universita' di Roma 2 Tor Vergata, Via della Ricerca Scientifica, 00133 Roma, Italy

E-mail address: trapani@mat.uniroma2.it 